\title{
Severe COVID-19 during Pregnancy and Possible Vertical Transmission
}

\author{
Maria Claudia Alzamora, MD ${ }^{1}$ Tania Paredes, MD² \\ Luis M. Valdez, MD ${ }^{5,6}$ Mauricio La Rosa, MD ${ }^{1,7}$ \\ 5 Tropical Medicine Institute "Alexander von Humbolt," Universidad \\ Peruana Cayetano Heredia, Lima, Peru \\ 6 Division of Infectious Disease, British American Hospital, Lima, Peru \\ ${ }^{7}$ Division of Maternal Fetal Medicine, University of Texas Medical \\ Branch, Texas
}

David Caceres, $\mathrm{MD}^{3}$ Camille M. Webb, MD ${ }^{4,5}$

Address for correspondence Mauricio La Rosa, MD, Division of Maternal-Fetal Medicine, British American Hospital, Lima, Peru; Division of Maternal-Fetal Medicine, University of Texas Medical Branch, 301 University Boulevard, Galveston, TX 77550 (e-mail: malarosa@utmb.edu).
Am J Perinatol 2020;37:861-865.
Keywords

- COVID-19

- coronavirus

- neonate

- pregnancy
There are few cases of pregnant women with novel corona virus 2019 (COVID-19) in the literature, most of them with a mild illness course. There is limited evidence about in utero infection and early positive neonatal testing. A 41-year-old G3P2 with a history of previous cesarean deliveries and diabetes mellitus presented with a 4-day history of malaise, lowgrade fever, and progressive shortness of breath. A nasopharyngeal swab was positive for COVID-19, COVID-19 serology was negative. The patient developed respiratory failure requiring mechanical ventilation on day 5 of disease onset. The patient underwent a cesarean delivery, and neonatal isolation was implemented immediately after birth, without delayed cord clamping or skin-to-skin contact. The neonatal nasopharyngeal swab, 16 hours after delivery, was positive for severe acute respiratory syndromecoronavirus 2 (SARS-CoV-2) real-time polymerase chain reaction (RT-PCR), and immunoglobulin (Ig)-M and IgG for SARS-CoV-2 were negative. Maternal IgM and IgG were positive on postpartum day 4 (day 9 after symptom onset). We report a severe presentation of COVID-19 during pregnancy. To our knowledge, this is the earliest reported positive PCR in the neonate, raising the concern for vertical transmission. We suggest pregnant women should be considered as a high-risk group and minimize exposures for these reasons.

\section{Key Points}

- We report a severe presentation of COVID-19 in pregnancy requiring invasive ventilatory support.

- This is a case of positive RT-PCR in first day of life, suggesting possible vertical transmission.

- There were no detectable maternal antibodies for COVID-19 until after delivery.

Copyright $\odot 2020$ by Thieme Medical Publishers, Inc., 333 Seventh Avenue, New York, NY 10001, USA. Tel: +1(212) 760-0888.
DOI https://doi.org/ 10.1055/s-0040-1710050. ISSN 0735-1631. 
The novel coronavirus disease 2019 (COVID-19), caused by the severe acute respiratory syndrome-coronavirus-2 (SARS-CoV-2) is a highly infectious disease that was first described by Huang et al, ${ }^{1}$ whose report focused primarily on the main characteristics and outcomes of nonpregnant adults. The largest published series in pregnancy described 55 pregnant women infected with COVID-19, of whom only one had a severe presentation, and there were no deaths. ${ }^{2}$ There is still much unknown regarding the impact of the disease on pregnancy, differences in clinical course, and outcomes in this population, as well as the risk of vertical transmission.

Physiological changes during pregnancy, such as reduced functional residual volumes, diaphragm elevation, and altered cell immunity, lead to increased susceptibility to viral infections and worse outcomes. ${ }^{2}$ In SARS and Middle East respiratory syndrome (MERS), up to 35 and $41 \%$ of pregnant patients required mechanical ventilation, and mortality rates were as high as 18 and $25 \%$, respectively. ${ }^{2-4}$ In contrast, the majority of reported cases of COVID-19 infection in pregnant patients have shown a mild or asymptomatic course of the disease, with only few cases requiring intensive care unit (ICU) admission, and only a few reported cases requiring mechanical ventilation. ${ }^{2,3,5-7}$

To date, there is no concrete evidence of whether there is in utero transmission of SARS-CoV-2. Two cases of neonates with positive real-time polymerase chain reaction (RT-PCR) testing soon after delivery have been described. ${ }^{2,5}$ However, these cases lack essential clinical data and information regarding adequate isolation, which raises the possibility of infection in the neonatal period.

Herein, we describe a case of COVID-19 infection in a pregnant woman requiring mechanical ventilation and cesarean delivery, as well as a neonate with positive testing, 16 hours after birth.

\section{Case Report}

On March 29, 2020, a 41-year-old woman (gravida 3, para 2) was transferred to our institution at 33 weeks of gestation due to respiratory insufficiency. Her medical history was remarkable for two previous cesarean deliveries and diabetes mellitus. She was treated with metformin and insulin until the beginning of the current pregnancy. She received prenatal care at another institution.

The patient presented with a 4-day history of general malaise, fatigue, and low-grade fever, and later developed worsening shortness of breath, which prompted her to seek medical attention. She was admitted to an outside hospital and remained there for 3 days. She received a steroid course of lung maturity during her admission at the outside hospital. She was then transferred to our institution for further care. Of importance, her partner and two children were symptomatic in the 15 days prior to admission. The partner tested positive for RT-PCR SARS-CoV-2 while the patient was in the hospital. In the emergency department, the patient's pulse was 131 beats per minute, the respiratory rate 38 breaths per minute, and the oxygen saturation $99 \%$ with a
Table 1 Initial maternal laboratory results

\begin{tabular}{|c|c|c|}
\hline Variable & Reference range & Result \\
\hline Hemoglobin (g/dL) & $12.3-15.3$ & 10.0 \\
\hline White-cell count (per $\mathrm{mm}^{3}$ ) & $4,400-11,300$ & 3,590 \\
\hline Platelet count (per $\mathrm{mm}^{3}$ ) & $149,000-409,000$ & 116,000 \\
\hline $\begin{array}{l}\text { Absolute lymphocyte count } \\
\text { (per } \mathrm{mm}^{3} \text { ) }\end{array}$ & $1,000-4,000$ & 467 \\
\hline C-reactive protein (mg/dL) & $0.00-0.50$ & 14.28 \\
\hline Procalcitonin ng/mL & $<0.50$ & 0.35 \\
\hline D-dimer $(\mu \mathrm{g} / \mathrm{mL})$ & $0.00-0.50$ & 0.79 \\
\hline Ferritin $(\mathrm{ng} / \mathrm{mL})$ & $15.0-150.0$ & 266.70 \\
\hline CPK (U/L) & $26.0-155.0$ & 17.0 \\
\hline Glucose (mg/dL) & $70.0-100.0$ & 105.0 \\
\hline Creatinine (mg/dL) & $0.5-1.20$ & 0.31 \\
\hline Blood urea nitrogen (mg/dL) & $5.13-23.3$ & 5.6 \\
\hline Total bilirubin (mg/dL) & $0.00-1.20$ & 0.41 \\
\hline $\begin{array}{l}\text { Aspartate aminotransferase } \\
(\mathrm{U} / \mathrm{L})\end{array}$ & $0.0-40.0$ & 18.0 \\
\hline $\begin{array}{l}\text { Alanine aminotransferase } \\
(\mathrm{U} / \mathrm{L})\end{array}$ & $0.0-38.0$ & 15.0 \\
\hline Sodium (mEq/L) & $135.0-145.0$ & 135.3 \\
\hline Potassium (mEq/L) & $3.5-5.5$ & 3.68 \\
\hline Chloride (mEq/L) & $97.0-111.0$ & 101.8 \\
\hline \multicolumn{3}{|l|}{ Arterial blood gases } \\
\hline $\mathrm{pH}$ & $7.35-7.43$ & 7.43 \\
\hline $\mathrm{PCO}_{2}$ & $32-43$ & 23 \\
\hline $\mathrm{PO}_{2}$ & $80-100$ & 196 \\
\hline $\mathrm{HCO}_{3}$ & $22.0-28.0$ & 15.3 \\
\hline $\mathrm{BE}$ & -2.0 to 2.0 & -7.7 \\
\hline
\end{tabular}

Abbreviations: BE: base excess; CPK, creatine phosphokinase.

FiO2 of approximately $90 \%$. Her body mass index (BMI) was $35 \mathrm{~kg} / \mathrm{m}^{2}$. Laboratory tests showed metabolic acidosis on arterial blood gases, pancytopenia, elevated C-reactive protein, elevated ferritin, and slightly elevated D-dimer and glucose ( - Table 1 ). A nasopharyngeal swab was obtained for SARS-CoV-2 RT-PCR which was reported as positive on the next day, and for Viral Filmarray and PneumoVir panel, both reported negative for common viruses. Initial tomography is shown in - Fig. 1.

The patient was intubated and placed on mechanical ventilation due to severe respiratory insufficiency in the setting of suspected COVID-19. Patient was started on azithromycin, hydroxychloroquine, meropenem, vancomycin, and oseltamivir. The patient underwent a cesarean delivery due to maternal respiratory compromised status. The neonate weighed 2,970 g, with Apgar's scores of 6 and 8 at 1 and 5 minutes, respectively. The neonate was intubated in another room due to the high level of sedation of the mother. Delayed cord clamping or skin-to-skin contact was not performed due to general depressed state of the neonate. He was immediately separated from his mother and was not exposed to family members, who were at home under strict 


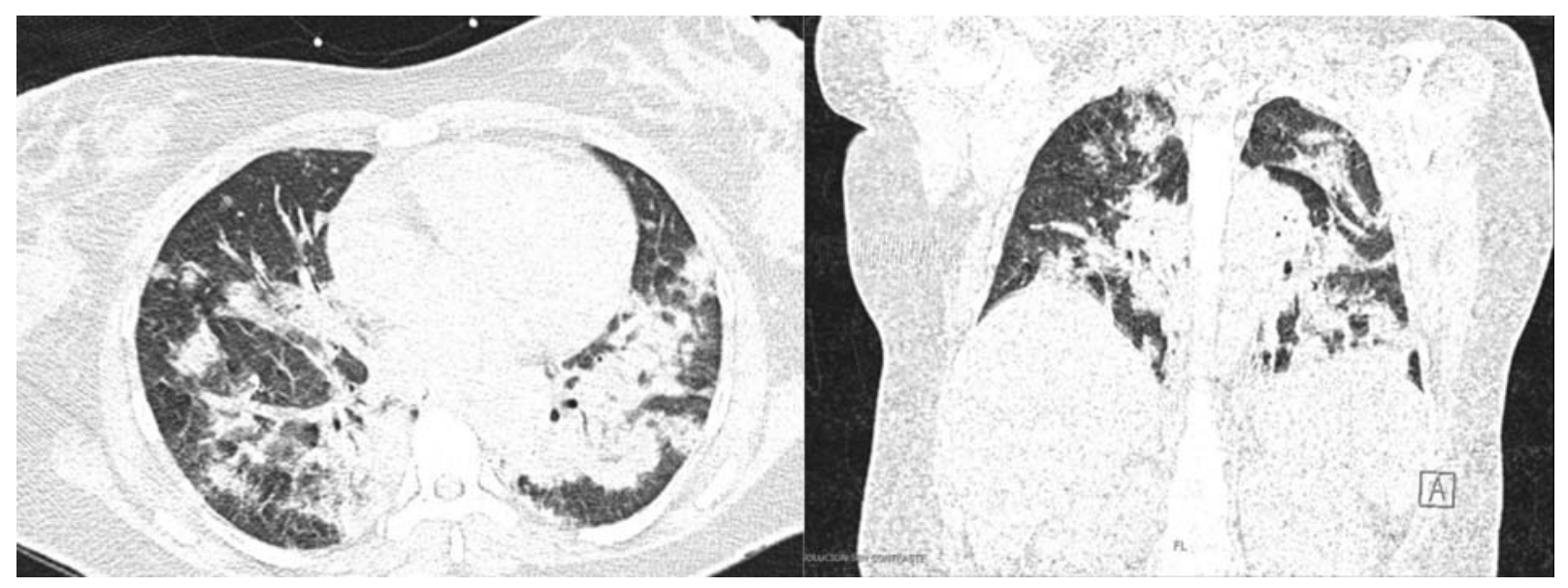

Fig. 1 CT scan of the chest showing multiple consolidations in both lungs, predominantly in bases and associated with bilateral pleural effusion. $\mathrm{CT}$, computed tomography.

isolation measures. Due to the maternal condition, maternal medical regimen, breastfeeding was not initiated. He was placed in the neonatal intensive care unit (NICU) with no other COVID-19 cases, as this was the first pediatric case at our institution. Chest X-ray showed no abnormalities.

Baseline and follow-up neonatal serology obtained at birth, and analyzed by solid-phase immunochromatographic assay (Zhejiang Orient Gene Biotech Co.), yielded negative immunoglobulin (Ig)-G and IgM titers. Nasopharyngeal swab of neonate for SARS-CoV-2 RT-PCR, obtained 16 hours after delivery (as soon as the maternal PCR resulted), was reported positive. RT-PCR repeated 48 hours later for confirmation was also positive. Maternal serology on postpartum day 1 showed negative IgG and IgM levels, followed by positive IgG and IgM on repeat testing on postpartum day 4 (day 9 after symptom onset).

The newborn required ventilatory support for 12 hours, after which he was extubated and placed on continuous positive airway pressure, with favorable outcome and not requiring antibiotic treatment. At the sixth day of life, the newborn presented mild respiratory difficulty and sporadic cough requiring supplemental oxygen with nasal cannula. Imaging and laboratory testing remain normal.

\section{Discussion}

We report a severe clinical presentation of COVID-19 during pregnancy, leading to respiratory failure, mechanical ventilation, and preterm delivery. This adds to a growing body of evidence which raises concerns about possible worse maternal outcomes in pregnancy, as seen with previous COVID-19 outbreaks. ${ }^{4,5}$ The most commonly described course of disease in pregnancy is that of an asymptomatic infection or mild pneumonia, and most cases resolved without undergoing delivery. ${ }^{2,3,5}$ Few cases required ICU admission and one case required invasive ventilation with extracorporeal membrane oxygenation. ${ }^{2,7}$ It is unknown whether pregnancy-related immune regulation alters the disease course by suppressing the exaggerated inflammatory response seen in this disease and associated with a poorer prognosis. ${ }^{8}$ It is important to mention that this patient had diabetes and class II obesity, both risk factors for severe COVID-19. We do not have enough reported data to ascertain that pregnancy does not lead to worse outcomes, particularly in high-risk pregnant patients. ${ }^{9,10}$

In previous reports, most of the deliveries were performed by cesarean. In our patient, a preterm cesarean delivery was performed due to her history of two prior cesareans, completed antenatal steroid course, and severe maternal pulmonary insufficiency. Different recommendations for cesarean delivery during COVID-19 infection have been described, most of them related to common obstetric factors such as history of cesareans, preeclampsia or fetal distress. There is a lack of convincing evidence that cesarean delivery is protective. Vaginal delivery is not contraindicated in patients with COVID-19. 2,11

The risk of vertical transmission remains unknown. A major finding in this case is the positive testing on RT-PCR of neonatal nasopharyngeal swab as soon as 16 hours after delivery. We believe there is a low probability of infection during the cesarean or postnatally due to sterility of the procedure and isolation measures implemented immediately after birth, strongly raising the suspicion of in utero transmission of SARS-CoV-2. Prior studies have failed to demonstrate maternal-fetal transmission of SARS-CoV-2, including negative testing in amniotic fluid, umbilical cord blood, vaginal swabs, and breast milk. ${ }^{2,5,11-13} \mathrm{~A}$ case series of nine pregnant women with COVID-19 demonstrated negative nasopharyngeal swabs on all neonates. Two neonates with positive RT-PCR testing as early as 30 hours after delivery have been reported; however, these cases lacked sufficient clinical data or precise information regarding isolation methods, and perinatal transmission could not be ruled out. ${ }^{2,5}$

Recently, Dong et al and Zeng et al reported virus-specific IgM antibodies in neonatal blood immediately after birth, with negative testing on RT-PCR of nasopharyngeal swab, in neonates with SARS-CoV-2 infected mothers. ${ }^{14,15}$ Given that IgM does not usually cross the placental barrier due to its structure, it is possible that IgM was produced in the fetus in response to vertical transmission of the virus. However this is not conclusive evidence, and may also be due to placental alterations allowing the passage of IgM, or false positive testing. 




Fig. 2 Timeline illustrating serologic assay results evolution in mother and neonate. Ig, immunoglobulin; RT-PCR, real-time polymerase chain reaction.

We found negative serology in both mother and neonate on the day of birth, and later seroconversion of the mother, likely due to the acute presentation of COVID-19. These are consistent with the serological conversion curve described in other studies in which IgM seroconverts after day 5 of symptom onset. The evolution of serologic testing of both the mother and neonate are shown in - Fig. 2. The neonatal serology remained negative up to 5 days of life. This could be explained by the immaturity of the adaptive immunity in the neonatal period, especially seen in preterm neonates, which results in impaired cytokine and B-cell immunoglobulin production relative to adults. ${ }^{16}$

Angiotensin-converting enzyme 2 (ACE2) has been described as a SARS-CoV-2 receptor in humans. There is conflicting evidence regarding the interaction of ACE2 and SARS$\mathrm{CoV}-2$ at the placental level. Dong et $\mathrm{al}^{14}$ demonstrated transient ACE2 overexpression and increased activity in the placenta and reproductive organs in rats during pregnancy. In contrast, Zeng et al ${ }^{15}$ described very low expression of ACE2 in almost all human cell types of the early maternalfetal interface, suggesting the placenta had virtually no susceptible cells to the virus.

\section{Limitations}

Our report has some limitations. The lag-time to neonatal nasopharyngeal swab, due to the current national criteria for testing, raises the possibility of perinatal transmission, though this was minimized by the procedures described above. We did not evaluate the presence of virus in amniotic fluid, cord blood, or placental tissue that could further clarify pathogenesis.

\section{Conclusion}

We describe a severe maternal presentation of COVID-19 during the third trimester of pregnancy, leading to mechanical ventilation and preterm delivery, as well as positive RTPCR, in the neonate. We believe these findings have important public implications both due to the severity of disease presentation, and the concern for vertical transmission. Given this information, pregnant women should be considered a vulnerable population in which exposure is to be avoided.

\section{Funding}

None.

\section{Conflict of Interest \\ None declared.}

\section{Acknowledgments}

We thank Dr. Luciano Poletti for the attainment of serologic testing in maternal and neonatal samples during the course of the study, Dr. Maria Mur for the clinical care of the neonate in the Pediatric Intensive Care Unit, Dr. Jorge Arias for his support in the obstetric clinical care, and the patient's family who gave consent for this case to be published.

\section{References}

1 Huang C, Wang Y, Li X, et al. Clinical features of patients infected with 2019 novel coronavirus in Wuhan, China. Lancet 2020;395 (10223):497-506

2 Dashraath P, Jing Lin Jeslyn W, Mei Xian Karen L, et al. Coronavirus Disease 2019 (COVID-19) Pandemic and Pregnancy. Am J Obstet Gynecol 2020;0(00):S0002-9378(20)30343-4

3 Liu D, Li L, Wu X, et al. pregnancy and perinatal outcomes of women with coronavirus disease (COVID-19) pneumonia: a preliminary analysis. Am J Roentgenol 2020 (e-pub ahead of print). Doi: 10.2214/AJR.20.23072

4 Schwartz DA, Graham AL. Potential maternal and infant outcomes from (Wuhan) coronavirus 2019-nCoV infecting pregnant women: lessons from SARS, MERS, and other human coronavirus infections. Viruses 2020;12(02):194

5 Chen H, Guo J, Wang C, et al. Clinical characteristics and intrauterine vertical transmission potential of COVID-19 infection in nine pregnant women: a retrospective review of medical records. Lancet 2020;395(10226):809-815

6 Iqbal SN, Overcash R, Mokhtari N, et al. An uncomplicated delivery in a patient with COVID-19 in the United States. N Engl J Med 2020. Doi: 10.1056/NEJMc2007605

7 Breslin N, Baptiste C, Miller R, et al. COVID-19 in pregnancy: early lessons. Am J Obstet Gynecol MFM 2020 (e-pub ahead of print). Doi: 10.1016/j.ajogmf.2020.100111

8 Vincenzo B. Coronavirus disease 2019 (COVID-19): Pregnancy issues. Available at: https://www.uptodate.com/contents/coronavirus-disease-2019-covid-19-pregnancy-issues? search=covid\%20pregnancy\&source $=$ search_result\&selectedTitle $=2 \sim 150 \&$ usage_type $=$ de fault\&display_rank=2\#H3682062658. Accessed April 04, 2020

9 Zhou F, Yu T, Du R, et al. Clinical course and risk factors for mortality of adult inpatients with COVID-19 in Wuhan, China: a retrospective cohort study. Lancet 2020;395(10229):1054-1062

10 Peng YD, Meng K, Guan HQ et al. [Clinical characteristics and outcomes of 112 cardiovascular disease patients infected by 2019-nCoV] (in Chinese). Zhonghua Xin Xue Guan Bing Za Zhi 2020;48:E004 (e-pub ahead of print). Doi: 10.3760/cma.j. cn112148-20200220-00105

11 Fan C, Lei D, Fang C, et al. Perinatal transmission of COVID-19 associated SARS-CoV-2: should we worry? Clin Infect Dis 2020 (epub ahead of print). Doi: 10.1093/cid/ciaa226 
12 Yang H, Wang C, Poon LC. Novel coronavirus infection and pregnancy. Ultrasound Obstet Gynecol 2020;55(04):435-437

13 Li Y, Zhao R, Zheng S, et al. Early release - lack of vertical transmission of severe acute respiratory syndrome coronavirus 2 , China. Emerging Infectious Diseases 2020;26(06). Doi: 10.3201/eid2606.200287

14 Dong L, Tian J, He S, et al. Possible vertical transmission of SARSCoV-2 from an infected mother to her newborn. JAMA 2020 (epub ahead of print). Doi: 10.1001/jama.2020.4621
15 Zeng H, Xu C, Fan J, et al. Antibodies in infants born to mothers with COVID-19 pneumonia. JAMA 2020 (e-pub ahead of print). Doi: $10.1001 /$ jama.2020.4861

16 Melville JM, Moss TJM. The immune consequences of preterm birth. Front Neurosci 2013;7:79. Available at: https://www.ncbi. nlm.nih.gov/pmc/articles/PMC365928. Accessed April 14, 2020 